\title{
Simulações de transporte reativo em um aquífero contaminado por querosene de aviação
}

\author{
Elias Hideo Teramoto* \\ Hung Kiang Chang \\ Marcus Paulus Martins Baessa \\ Adriana Soriano ${ }^{2}$ \\ ${ }^{1}$ Universidade Estadual Paulista Júlio de \\ Mesquita Filho \\ Instituto de Geociências e Ciências Exatas \\ de Rio Claro \\ Departamento de Geologia Aplicada \\ Av. 24 A, 1515 \\ Bela Vista \\ Rio Claro SP Brasi \\ 13506900 \\ * Autor correspondente \\ elias.hideo-teramoto@unesp.br
}

\begin{abstract}
RESUMO
A capacidade de bactérias nativas em biodegradar os hidrocarbonetos em sub-superfície têm sido estudados de maneira consistente desde a década de 1970 , tendo em vista que esta classe de contaminantes pode oferecer severos riscos à saúde humana. $\mathrm{O}$ entendimento dos mecanismos que governam a biodegradação de hidrocarbonetos é crucial para o gerenciamento de áreas contaminadas. Por esta razão, simulações numéricas de transporte reativo foram realizadas para avaliar modelo geoquímico conceitual e auxiliar no entendimento dos mecanismos que governam a biodegradação de BTEX em um aquífero laterítico contaminado por grande volume de combustível de aviação. O modelo conceitual previamente proposto para associar o zoneamento geoquímico com a mineralização BTEX e reações secundárias foi testado por intermédio de modelos de transporte reativos. A representação da especiação química simulada do aquífero contaminado com hidrocarbonetos estudado é melhor suportada quando a $\mathrm{PCO}_{2}$ é fixada e o oxigênio dissolvido, potencialmente presente devido à transferência de massa do ar trapeado, é considerado no modelo. Estes resultados sugerem a necessidade de ambas as restrições serem consideradas nos modelos de transporte reativo envolvendo biodegradação de hidrocarbonetos.
\end{abstract}

Palavras-chave: $\mathrm{PCO}_{2}$, Aquífero laterítico, Modelo de transporte reativo, Redução de ferro, Metanogênese.

\section{ABSTRACT}

The ability of native bacteria to biodegrade subsurface hydrocarbons has been studied consistently since the 1970s, since this class of contaminants may pose severe human health risks. Understanding the mechanisms that govern hydrocarbons biodegradation represents a crucial issue for the management of contaminated areas. For this reason, numerical simulations of reactive transport were performed to evaluate the conceptual geochemical model and to helpunderstand the mechanisms that govern BTEX biodegradation in a lateritic aquifer contaminated by large aviation fuel. The feasibility of previously proposed conceptual model linking geochemical zonation with BTEX mineralization and secondary reactions was evaluated by means of reactive transport models. The representation of the simulated chemical speciation of the studied hydrocarbon-contaminated aquifer is best supported when $\mathrm{PCO}_{2}$ is fixed and dissolved oxygen, potentially present due to mass transfer from entrapped air, is considered in the model. These findings suggest the need for both constraints to be considered in the reactive transport models involving hydrocarbon biodegradation.

Keywords: $\mathrm{PCO}_{2}$, Lateritic aquifer, reactive transport model, iron reduction, methanogenesis.

\section{INTRODUÇÃO}

Em razão do grande volume de combustíveis derivados de petróleo que são produzidos, estocados ou transferidos, vazamentos de combustíveis são cenários comuns de contaminação subsuperficial em refinarias. Ao ser acidentalmente liberado no solo, os hidrocarbonetos migram até atingir as vizinhanças da zona saturada, onde passam a 
se acumular, distribuindo- se como LNAPL (fase líquida não aquosa). A flutuação do nível d'água promove o aprisionamento do LNAPL por capilaridade na zona saturada, fenômeno conhecido por trapeamento, fazendo com que este perca mobilidade. A presença de LNAPL trapeado na porção saturada de um aquífero é responsável pela liberação de compostos solúveis para a água, em resposta a mecanismos de transferência de massa (MILLER et al., 1990). Dentre os compostos solubilizados em água merece destaque o BTEX (benzeno, tolueno, etilbenzeno $\mathrm{e}$ xilenos), em razão de sua solubilidade e dos riscos associados à saúde humana.

Décadas de estudos laboratoriais e de campo forneceram evidências sólidas de que a ação metabólica de bactérias nativas é responsável pela metabolização de hidrocarbonetos solubilizados sob condições aeróbicas e anaeróbicas (WIEDEMEIER et al. 1999; NATIONAL RESEARCH COUNCIL, 2000; DECLERCQ et al., 2012). Em casos nos quais uma quantidade suficiente de receptores de elétrons está disponível, a biodegradação de hidrocarbonetos solubilizados pode ocorrer rapidamente (WIEDEMEIER et al. 1999).

Os modelos de transporte reativo representam ferramentas úteis que permitem a reprodução da complexa sobreposição de vários processos biogeoquímicos relacionados à mineralização do BTEX, permitindo cálculos de balanço de massa em cenários complexos (MAYER et al., 2002). A simulação geoquímica de aquíferos contaminados por compostos BTEX é mediada pela solução numérica do conjunto de reações químicas e dos estados de equilíbrio dos subprodutos orgânicos $\mathrm{e}$ inorgânicos diretamente associadas à biodegradação de hidrocarbonetos na fase

\section{MATERIAIS E MÉTODOS}

\section{1. ÁREA DE ESTUDO}

O local do estudo situa-se no município de Paulínia (SP), onde uma grande quantidade de combustível de aviação está presente em subsuperfície, com um volume estimado de $520 \mathrm{~m}^{3}$ (PEDE, 2009). A contaminação foi identificada durante uma investigação no site em 2002, e um processo de remediação ativa, pela técnica de bombeamento e tratamento, está em operação desde 2005. Para determinar o aquosa (HUNTER et al., 1998, MAYER et al., 2002).

Em aquíferos onde se verifica a abundância de óxidos de ferro no sedimento, a redução do $\mathrm{Fe}(\mathrm{III})$ é a principal rota metabólica para a biodegradação de hidrocarbonetos. Diversos modelos conceituais e modelos reativos com características particulares, dotados de abordagens numéricas e condicionantes geoquímicos distintos, foram propostos para reproduzir a mineralização de BTEX via redução de ferro, destacando-se Prommer et al. (1999), Schreiber et al. (2004), Vencelides et al. (2007), Miles et al. (2008), Colombani et al. (2009) e $\mathrm{Ng}$ et al. (2015). Entretanto, nenhum desses trabalhos foi conduzido em climas tropicais ou subtropicais, onde prevalecem condicionantes geoquímicos $\mathrm{e}$ hidrológicos específicos.

A carência do entendimento das condicionantes geoquímicas típicas de climas tropicais se deve ao fato da ampla maioria de estudos relacionados à mineralização de hidrocarbonetos terem sido conduzidos na Europa e na América do Norte, onde prevalece clima do tipo temperado. Desse modo, para subsidiar a correta interpretação de dados geoquímicos monitorados em climas tropicais, deve-se propor um modelo conceitual que contemple as características específicas desse tipo climático. Teramoto e Chang (2019) elaboraram um modelo geoquímico conceitual para explicar as reações associadas à biodegradação de hidrocarbonetos em aquíferos situados em climas subtropicais e tropicais. Com vistas a estimar a adequabilidade deste modelo conceitual, o presente trabalho apresenta os resultados de simulações de transporte reativo, avaliando a aderência dos resultados das simulações com dados de campo. limite da migração do LNAPL e das plumas da fase dissolvida dos compostos BTEX, foram instalados 117 poços de monitoramento em uma área de $264.600 \mathrm{~m}^{2}$. A Figura 1 mostra o mapa potenciométrico, o limite da área-fonte e a localização dos poços de monitoramento e bombeamento na área de estudo. A áreafonte ocupava uma área de aproximadamente $80.000 \mathrm{~m}^{2}$. 


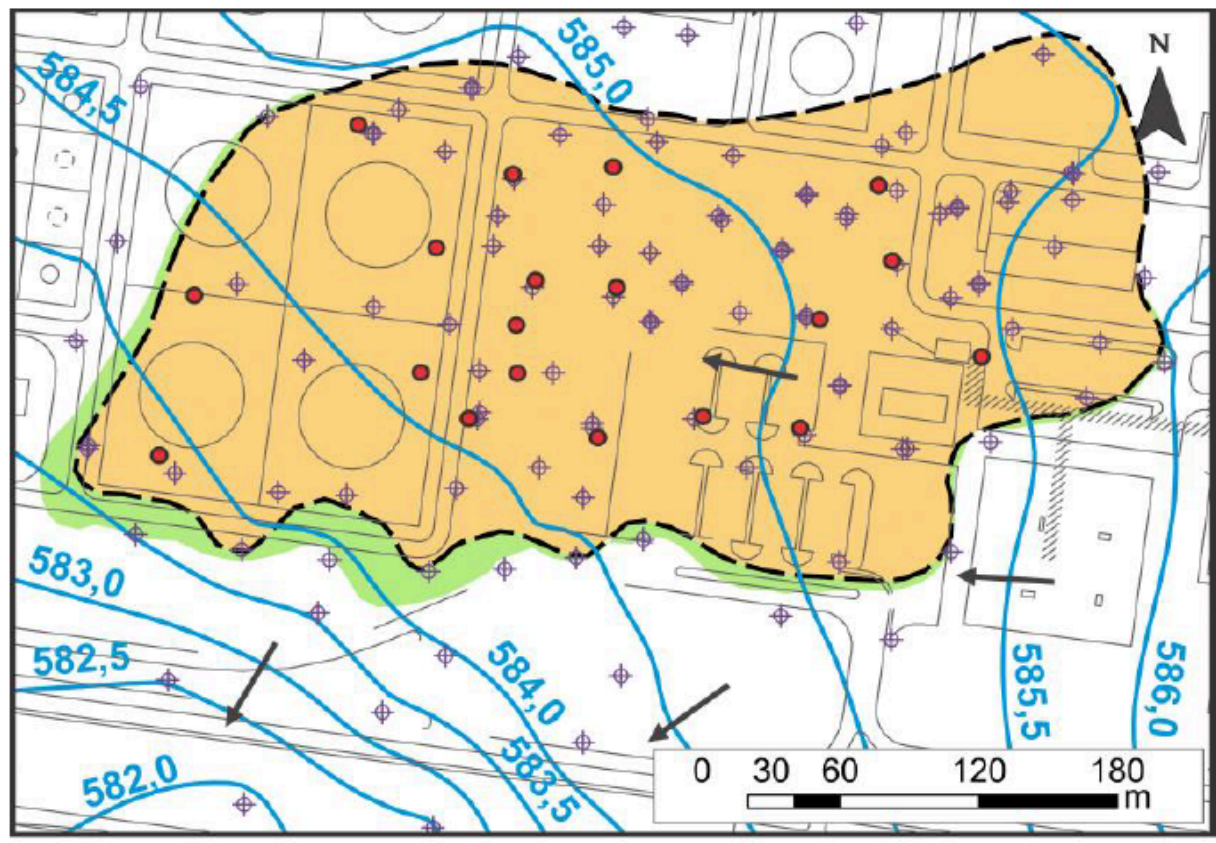

Legenda

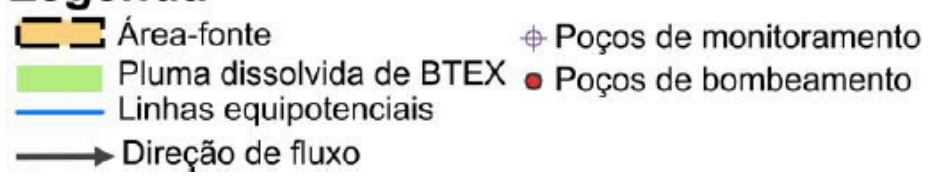

Figura 1

Localização dos poços de monitoramento e bombeamento na área de estudo e os limites da área-fonte (TERAMOTO; CHANG, 2017).

O aquífero raso existente no local, é composto por sedimentos cenozoicos texturalmente heterogêneos, sendo majoritariamente representados por areias médias a grossas argilosas, areias grossas microconglomeráticas, argilas arenosas e siltes argilosos (BORDIGNON et al., 2015). A geometria das fácies arenosas e argilosas indica ambiente deposicional de rios meandrantes, com predomínio de depósitos de planícies de

\subsection{MODELO GEOQUÍMICO CONCEITUAL}

Com base no conjunto de dados coletados durante as sucessivas investigações e monitoramento da qualidade da água na área, Teramoto e Chang (2019) propuseram um modelo conceitual para explicar o efeito de inundação.

Os valores de condutividades hidráulicas, determinados por testes de slug realizados em 64 poços de monitoramento, variam de 1,2 X $10^{-7} \mathrm{~m} / \mathrm{s}$ a $2,4 \times 10^{-4} \mathrm{~m} / \mathrm{s}$, com média geométrica de $2,8 \times 10^{-5} \mathrm{~m} / \mathrm{s}$ (TERAMOTO; CHANG, 2017). O gradiente hidráulico médio é de 0,0036 na porção nordeste da área e aumenta para sudoeste, em direção à zona de descarga, onde alcança o valor de 0,0176.

\subsubsection{LIBERAÇÃO DE COMPOSTOS BTEX PARA A ÁGUA}

A ampla variação da flutuação do nível d'água cria uma zona com LNAPL trapeado na maior parte do tempo na zona saturada (TERAMOTO; CHANG, 2017). Devido às condições de trapeamento, o mecanismo mais importante que opera na depleção de BTEX do LNAPL é representado pela transferência de massa para as águas subterrâneas. A dissolução do LNAPL ocorre quando o lençol freático limpo libera os poros da área-fonte. $\mathrm{O}$ movimento de compostos solúveis do LNAPL para a água é mediado pela transferência de 
massa de taxa limitada, podendo ser calculado pela Equação 1 (MILLER et al., 1990):

$$
J=\frac{d C_{a}}{d t}=k_{a} a_{n w}\left(C_{s}-C\right)
$$

Onde $\mathrm{J}=$ fluxo de massa dos hidrocarbonetos residuais para água $\left(\mathrm{ML}^{-2} \mathrm{~T}^{-1}\right)$, $k_{a}=$ coeficiente de transferência de massa $\left(\mathrm{LT}^{-1}\right), C_{s}=$ concentração aquosa máxima de um composto solúvel $\left(\mathrm{ML}^{-3}\right), \quad C=$ concentração aquosa de um composto solúvel $\left(\mathrm{ML}^{-3}\right)$ e $a_{w}=$ área da interface entre água e $\operatorname{NAPL}\left(\mathrm{L}^{2}\right)$.

\subsubsection{CONDICIONANTES GEOQUÍMICOS}

O cálculo de especiação da água subterrânea natural foi realizado em condição de equilíbrio químico, com pressão parcial de $\mathrm{CO}_{2}\left(\mathrm{PCO}_{2}\right)$ de $10^{-1,5} \mathrm{~atm}$, responsável pela manutenção de um valor de $\mathrm{pH}$ de aproximadamente 4,7 e um valor de alcalinidade próximo de $1 \mathrm{mg} / \mathrm{L}$, que são semelhantes aos das medições de campo. Os valores de $\mathrm{pH}$ medidos na área- fonte são próximos de 6 e caem gradualmente para jusante. A biodegradação sob redução de $\mathrm{Fe}$ (III) explica o forte aumento do $\mathrm{pH}$ na área-
Teramoto e Chang (2017) mostraram que a saturação do LNAPL é maior no centro da área-fonte e diminui em direção às bordas. Uma vez que a depleção de LNAPL em relação aos compostos BTEX está relacionada à saturação do LNAPL, a maior concentração, tanto da fração molar de BTEX no LNAPL quanto da solubilidade efetiva de BTEX nas águas subterrâneas, é observada na porção central da área-fonte. Devido à variabilidade na solubilidade efetiva, o BTEX dissolvido na fase aquosa abrange uma ampla gama de concentrações $(0,023 \mathrm{mg} / \mathrm{L}$ a $5,34 \mathrm{mg} / \mathrm{L})$.

fonte, uma vez que o $\mathrm{pH}$ está relacionado à concentração de $\mathrm{HCO}_{3}^{-}$

De acordo com Teramoto e Chang (2019), a flutuação do nível d'água promove o trapeamento do ar na zona saturada, que passa a liberar oxigênio para a água por difusão. Em razão disso, ocorrem condições favoráveis para elevação do potencial redox da água e oxidação das espécies químicas reduzidas $\left(\mathrm{Fe}^{2+} \mathrm{e}_{4}\right)$, produzidas pela biodegradação de hidrocarbonetos.

\subsubsection{REAÇÕES QUÍMICAS ASSOCIADAS À BIODEGRADAÇÃO DE HIDROCARBONETOS}

Devido à abundância de ferro, típica de aquíferos lateríticos em ambientes tropicais, a biodegradação via redução de ferro é um importante mecanismo para a mineralização de
BTEX em subsuperfície. Segundo Teramoto e Chang (2019), a reação de mineralização do tolueno incorpora o efeito da $\mathrm{PCO}_{2}$, conforme apresentado na Equação 2.

$$
\mathrm{C}_{7} \mathrm{H}_{8}+36 \mathrm{FeOOH}+14 \mathrm{H}_{2} \mathrm{O}+65 \mathrm{CO}_{2} \rightarrow 72 \mathrm{HCO}_{3}^{-}+36 \mathrm{Fe}^{2+}
$$

Vários estudos, como os de Schreiber et al. (2004) e Miles et al. (2008), demonstraram que a biodegradação sob redução de $\mathrm{Fe}$ (III) e metanogênese geralmente ocorrem concomitantemente. A Equação 3 descreve a biodegradação do tolueno por metanogênese.

$$
\mathrm{C}_{7} \mathrm{H}_{8}+5 \mathrm{H}_{2} \mathrm{O} \rightarrow 4,5 \mathrm{CH}_{4}+2,5 \mathrm{CO}_{2}
$$

As concentrações de $\mathrm{Fe}^{2+}$ e $\mathrm{CH}_{4}$ são elevadas na área-fonte, suportando a hipótese que as vias de biodegradação de BTEX sob redução de ferro e metanogênese ocorrem aí simultaneamente.

Segundo Teramoto e Chang (2019), durante o movimento ascendente do nível d'água ocorre o trapeamento do ar, promovendo a oxigenação do aquífero. A transferência de massa entre o ar trapeado e a água subterrânea fornece oxigênio à água, promovendo a oxidação dos subprodutos. $\mathrm{Na}$ área-fonte, o oxigênio liberado do ar trapeado rapidamente promove a oxidação do $\mathrm{Fe}^{2+}$, mantendo o gradiente de concentração elevado. $\mathrm{O} \mathrm{Fe}^{2+}$ oxidado precipita na fase instável de $\mathrm{Fe}(\mathrm{OH})_{3}$ (Eq. 4).

$$
\begin{gathered}
\mathrm{Fe}^{2+}+0,25 \mathrm{O}_{2}+\mathrm{HCO}_{3}^{-}+0,5 \mathrm{H}_{2} \mathrm{O} \rightarrow \mathrm{Fe}(\mathrm{OH})_{3}+2 \mathrm{CO}_{2} \\
\text { Geochimica Brasiliensis 33(3): } 260-272,2019
\end{gathered}
$$


De modo similar ao $\mathrm{Fe}^{2+}$, o $\mathrm{CH}_{4}$ também é oxidado pela catálise de bactérias nativas, empregando o oxigênio difundido do ar trapeado para a fase aquosa (Eq. 5).

$$
\mathrm{CH}_{4(a q)}+2 \mathrm{O}_{2} \rightarrow 2 \mathrm{H}_{2} \mathrm{O}+\mathrm{CO}_{2(a q)}
$$

As reações 4 e 5, conhecidas como reações secundárias, são normalmente associadas a ambiente oxidante. Entretanto, de acordo com as premissas apresentadas por Teramoto e Chang (2019), essas reações podem ocorrer na área-fonte, quando da presença de ar trapeado no meio poroso.

\subsubsection{ZONEAMENTO GEOQUÍMICO}

Em decorrência das equações 1 a 5, Teramoto e Chang (2019) admitem a existência de um zoneamento geoquímico, que é função da disponibilidade das espécies químicas envolvidas nessas reações, bem como em sua
Durante o período de estiagem a água subterrânea é drenada do espaço de poros e a maior parte da zona "quase saturada" desaparece, reduzindo assim criticamente a liberação de oxigênio para a zona saturada e, consequentemente, também as taxas de oxidação de subprodutos.
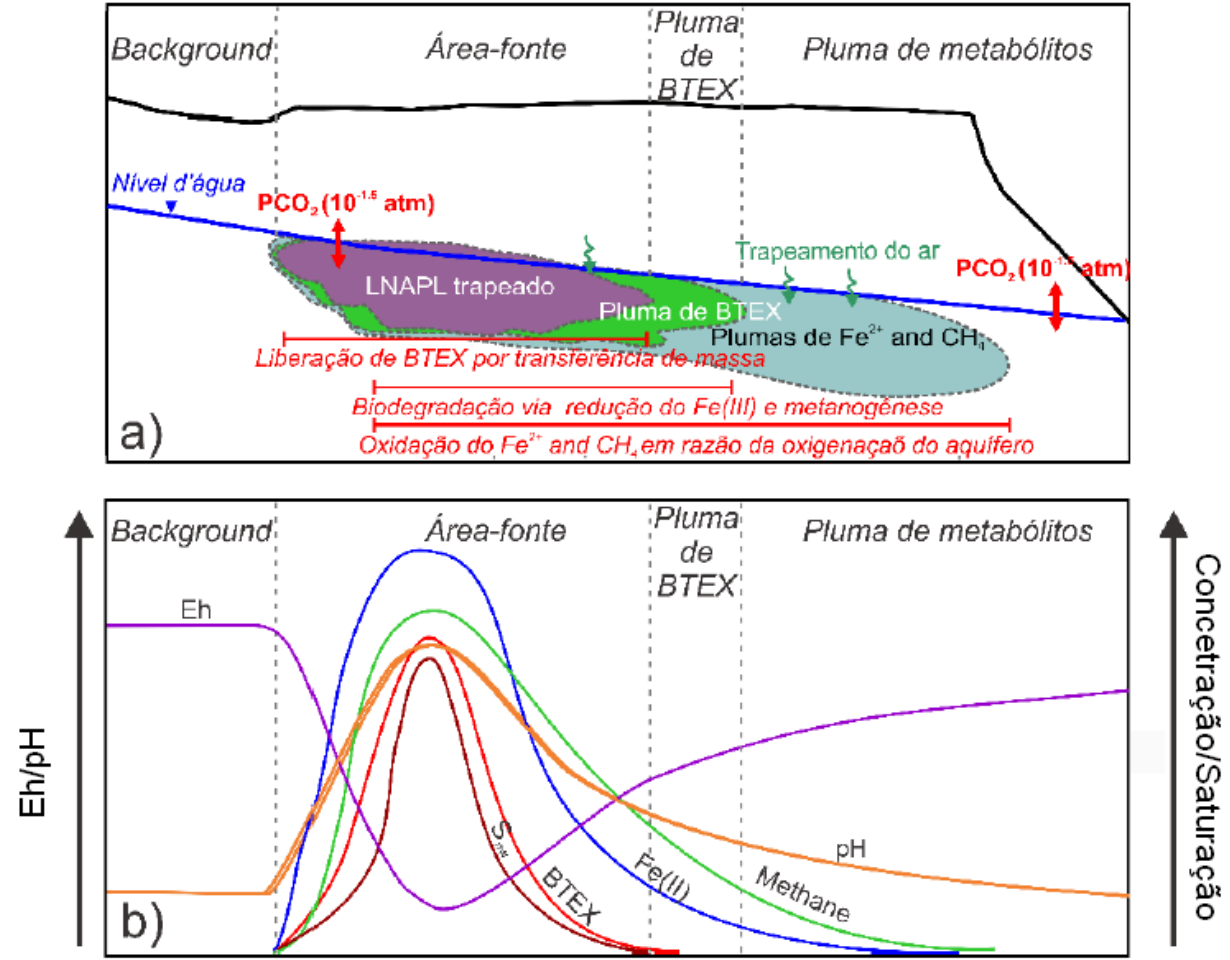

cinética. No modelo conceitual concebido pelos referidos autores existem quatro zonas geoquímicas distintas (Figura 2): (i) background, (ii) área-fonte, (iii) pluma BTEX e (iv) pluma de subprodutos.

O aquífero local é raso, tamponado por elevada $\mathrm{PCO}_{2}$ e de constituição mineralógica simples (quartzo, goetita, caulinita e muscovita); a água subterrânea em sua condição natural possui condutividade elétrica menor que $15 \mathrm{mS} / \mathrm{cm}$ e $\mathrm{pH}$ inferior a 4,8 (TERAMOTO; CHANG, 2019). Esta solução com baixa condutividade, $\mathrm{pH}$ reduzido e composição química próxima à da água de chuva caracteriza a zona geoquímica Background. A água isenta de contaminação flui para a área-fonte, onde ocorre a solubilização dos compostos BTEX por difusão. Devido à biodegradação do BTEX via redução de $\mathrm{Fe}$ (III) e metanogênese na área- fonte, observa-se um ambiente redutor com altas concentrações de $\mathrm{Fe}^{2+}$ e $\mathrm{CH} 4$. A quase totalidade dos compostos BTEX é biodegradada na área-fonte e apenas uma pequena fração é transportada para jusante, gerando as plumas de fase dissolvida desses compostos. 
$\mathrm{O} \mathrm{Fe}^{2+}$ como o $\mathrm{CH}_{4}$, produzidos na biodegradação dos compostos BTEX na áreafonte, são transportados por advecção para porções a jusante, gerando a pluma de metabólitos. Ao longo do fluxo, as concentrações de $\mathrm{Fe}^{2+}$ e $\mathrm{CH}_{4}$ passam a cair em razão da oxidação mediada por metabolismo microbiano. Como observado na Eq. 4, a oxidação do $\mathrm{Fe}^{2+}$ também reduz a alcalinidade, fazendo com que o pH e SI siderita (índice da saturação em relação à siderita) sejam igualmente reduzidos.

A Tabela 1 resume os valores médios dos parâmetros físico-químicos, BTEX e concentrações de $\mathrm{Fe}^{2+}$ e $\mathrm{CH}_{4}$ em cada uma das zonas geoquímicas propostas por Teramoto e Chang (2019).

Tabela 1 - Valores médios dos parâmetros geoquímicos mais relevantes das zonas geoquímicas (Teramoto; Chang, 2017).

\begin{tabular}{|c|c|c|c|c|c|c|c|c|}
\hline $\begin{array}{c}\text { Zona } \\
\text { Geoquímica }\end{array}$ & $\begin{array}{l}\text { BTEX } \\
(\mu \mathrm{g} / \mathrm{L})\end{array}$ & $\begin{array}{c}\mathrm{Fe}^{2+} \\
(\mathrm{mg} / \mathrm{L})\end{array}$ & $\begin{array}{c}\mathrm{CH}_{4} \\
(\mathrm{mg} / \mathrm{L})\end{array}$ & $\begin{array}{c}\text { Alcalinidade } \\
(\mathrm{mg} / \mathrm{L})\end{array}$ & pH & $\begin{array}{c}\mathbf{E C} \\
(\mathrm{mS} / \mathrm{cm})\end{array}$ & $\begin{array}{c}\text { Eh } \\
(\mathrm{mV})\end{array}$ & $\begin{array}{l}\text { Índice de saturação } \\
\text { da } \quad \text { Siderita } \log (Q / K)\end{array}$ \\
\hline Background & 0,000 & 0,023 & 0,002 & 6,33 & 4,71 & 11,37 & 281,50 & $<-6,00$ \\
\hline $\begin{array}{l}\text { Pluma de } \\
\text { metabólitos }\end{array}$ & 0,000 & 0,118 & 0,050 & 21,81 & 5,27 & 66,80 & 299,00 & $-5,38$ \\
\hline $\begin{array}{l}\text { Pluma de } \\
\text { BTEX }\end{array}$ & 22,26 & 12,052 & 1,94 & 39,17 & 5,47 & 89,36 & 130,80 & $-2,82$ \\
\hline Área-fonte & 1777,82 & 16,401 & 2,543 & 44,05 & 5,62 & 101,33 & $-120,04$ & $-1,82$ \\
\hline
\end{tabular}

\subsection{SIMULAÇÕES GEOQUÍMICAS}

Para confirmar se as reações presumidas no modelo conceitual são compatíveis com o zoneamento geoquímico conceitual, foram realizadas simulações empregando um modelo sintético bidimensional, por meio do programa $\mathrm{X} 2 \mathrm{t}$, do pacote Geochemist's Workbench (BETHKE; YEAKEL, 2016), que simula o transporte reativo empregando o método de diferenças finitas. $\mathrm{O}$ tempo simulado compreende um período de 3650 dias. O modelo sintético foi representado por um domínio retangular de $200 \mathrm{~m} \times 60 \mathrm{~m}$ em camada única, com espessura de $2 \mathrm{~m}$. A discretização do modelo baseou-se em uma malha de diferenças finitas composta de 3000 células, cada uma com $2 \mathrm{~m}^{2}$. Em relação às propriedades físicas do meio poroso, assumiuse velocidade de fluxo de $92,5 \mathrm{~m} / \mathrm{ano} \mathrm{e}$ porosidade efetiva de 0,22. O gradiente hidráulico imposto foi de 0,016 para reproduzir as condições de campo. A áreafonte foi idealizada como tendo $40 \mathrm{~m} \times 20 \mathrm{~m}$ x $2 \mathrm{~m}$ (comprimento $\mathrm{x}$ largura $\mathrm{x}$ altura), limitada nas bordas oeste e leste por condições de contorno do tipo Dirichlet (primeiro tipo), impondo um fluxo de leste para oeste. Assim, de forma reduzida, os valores de gradiente hidráulico, porosidade efetiva, condutividade hidráulica, dispersividade longitudinal e dispersividade transversal empregadas no modelo foram de $0,016,0,22,5 \times 10^{-5} \mathrm{~m} / \mathrm{s}, 1,2 \mathrm{~m}$ e $0,0001 \mathrm{~m}$, respectivamente.

A solução de entrada (Tabela 2) foi representada por uma solução igual ao Background (Tabela 1), impondo um equilíbrio químico com $\mathrm{PCO}_{2}$ de $10^{-1,5} \mathrm{~atm}$, representado pela fixação de uma concentração de $\mathrm{CO}_{2}$ aquoso de 47,28 mg/L. Para reproduzir a liberação dos compostos solúveis nas águas subterrâneas por transferência de massa interfásica (Eq. 1), 200 poços de injeção foram empregados para introduzir um volume constante de $100 \mathrm{mg}$ de tolueno por dia. Similarmente ao tolueno, para reproduzir a difusão de $\mathrm{O} 2$ para a água subterrânea, a partir do ar trapeado, foi imposta a injeção de 200 $\mathrm{mg}$ de oxigênio por dia via esses poços. Os íons $\mathrm{Na}^{+}$e $\mathrm{Cl}^{-}$foram inseridos no modelo apenas para fazer o balanço de cargas nas soluções simuladas. A Tabela 2 apresenta as soluções iniciais e de entrada empregadas nas simulaçõesrealizadas neste estudo.

A difusão de BTEX do LNAPL para a fase aquosa no espaço poroso é um mecanismo d taxa limitada, que restringe a disponibilidade do substrato nas águas subterrâneas e, consequentemente, também as taxas de biodegradação. Sob esse pressuposto, foi utilizado um modelo cinético de primeira ordem para governar as reações de biodegradação e outras reações oxi-redutoras nas simulações realizadas. 


\begin{tabular}{cc}
\hline Parâmetro & Solução inicial \\
\hline $\mathrm{pH}$ & 4 \\
Tolueno & $0 \mathrm{mg} / \mathrm{L}$ \\
$\mathrm{Fe}^{2+}$ & $0,023 \mathrm{mg} / \mathrm{L}$ \\
$\mathrm{HCO}_{3}^{-}$ & $1,6 \mathrm{mg} / \mathrm{L}$ \\
$\mathrm{CH}_{4(\mathrm{aq})}$ & $0,002 \mathrm{mg} / \mathrm{L}$ \\
$\mathrm{Na}^{+}$ & $1,3 \mathrm{mg} / \mathrm{L}$ \\
$\mathrm{Cl}^{-}$ & $2,0 \mathrm{mg} / \mathrm{L}$ \\
$\mathrm{O}_{2(\mathrm{aq})}$ & $8,2 \mathrm{mg} / \mathrm{L}$ \\
\hline
\end{tabular}

\section{RESULTADOS}

\subsection{SIMULAÇÕES NUMÉRICAS DE TRANSPORTE REATIVO}

\subsubsection{SIMULAÇÃO DO MODELO CONCEITUAL PROPOSTO}

Para testar a adequação do modelo conceitual sumariado na Figura 2, simulações numéricas foram realizadas impondo 0 conjunto de reações e restrições geoquímicas que definem o modelo. Os resultados fornecidos (Figura 3) pelas simulações reproduziram os valores de variação e as tendências espaciais observadas no campo em relação ao BTEX (representado pelo tolueno), $\mathrm{Fe}^{2+}, \mathrm{CH}_{4}, \mathrm{HCO}_{3}^{-}$, índice de saturação em relação à siderita, $\mathrm{pH}$ e potencial redox do par $\mathrm{Fe}^{3+} / \mathrm{Fe}^{2+}$
A semelhança entre os resultados da simulação (Figura 3) e as medições de campo (Tabela 2) indica que o modelo conceitual proposto por Teramoto e Chang (2019) é suficiente e adequado para explicar o zoneamento geoquímico da área de estudo. A $\mathrm{PCO}_{2}$ fixada e o efeito da oxigenação do aquífero são cruciais para assegurar a reprodutibilidade dos modelos de transporte reativo. A Tabela 3 apresenta os coeficientes de decaimento de primeira ordem empregados nas Eq. 2 a 5.

Tabela 3 - Taxas obtidas a partir de ajustes no modelo numérico

\begin{tabular}{cc}
\hline Reações & $\begin{array}{c}\text { Coeficiente de decaimento } \\
\text { de primeira ordem }\end{array}$ \\
\hline Biodegradação de BTEX via redução de Fe(III) (Eq. 2) & 0,0099 dias \\
Biodegradação de BTEX via metanogênese (Eq.3) & 0,0130 dias \\
Oxidação do Fe(II) (Eq. 4) & 0,0120 dias \\
Oxidação do metano (Eq. 5) & 0,024 dias \\
\hline
\end{tabular}

\subsubsection{SIMULAÇÃO DE CENÁRIOS ALTERNATIVOS}

Tendo sido verificada a viabilidade do modelo geoquímico conceitual proposto por Teramoto e Chang (2019) para explicar as condições geoquímicas observadas em campo, procedeu-se à investigação da importância relativa da $\mathrm{PCO}_{2} \mathrm{e}$ do ar trapeado na especiação química de aquíferos contaminados por hidrocarbonetos. Para este fim, foram simulados dois cenários geoquímicos alternativos à simulação inicial, ora referenciada como Cenário 1.

O primeiro cenário alternativo, Cenário 2, é semelhante ao Cenário 1, exceto pela eliminação da $\mathrm{PCO}_{2}$ fixada, permitindo que o $\mathrm{pH}, \quad$ a concentração de $\mathrm{HCO}_{3}^{-}$e, consequentemente, $\mathrm{O}$ SI da siderita variassem sem o efeito do tamponamento do $\mathrm{CO}_{2}$. No segundo cenário alternativo, Cenário 3 , o efeito da oxidação mediada pela dissolução do ar trapeado foi suprimido a partir da não representação das reações 4 e 5 nas simulações.

A Figura 4 apresenta os resultados dos três cenários reproduzidos ao longo de uma linha de fluxo na porção central do domínio do modelo sintético.

Como ilustrado na Figura 4, os Cenários 2 e 3 apresentam resultados fortemente dissimilares ao Cenário 1. As concentrações de $\mathrm{Fe}^{2+}$ e $\mathrm{CH}_{4}$ no Cenário 3 são maiores do que aquelas nos cenários 1 e 2 , e bastante distinta daquelas observadas em campo, apresentadas por Teramoto e Chang (2019).

$\mathrm{O} \mathrm{HCO}_{3}^{-}$é distinto nos três cenários simulados e indica que esta espécie química é sensível ao equilíbrio com $\mathrm{PCO}_{2}$ e reações de oxi-redução. No caso do Cenário 2, as concentrações de $\mathrm{HCO}_{3}^{-}$são menores devido à ausência de $\mathrm{CO}_{2}$ na fase aquosa para ser hidro- 

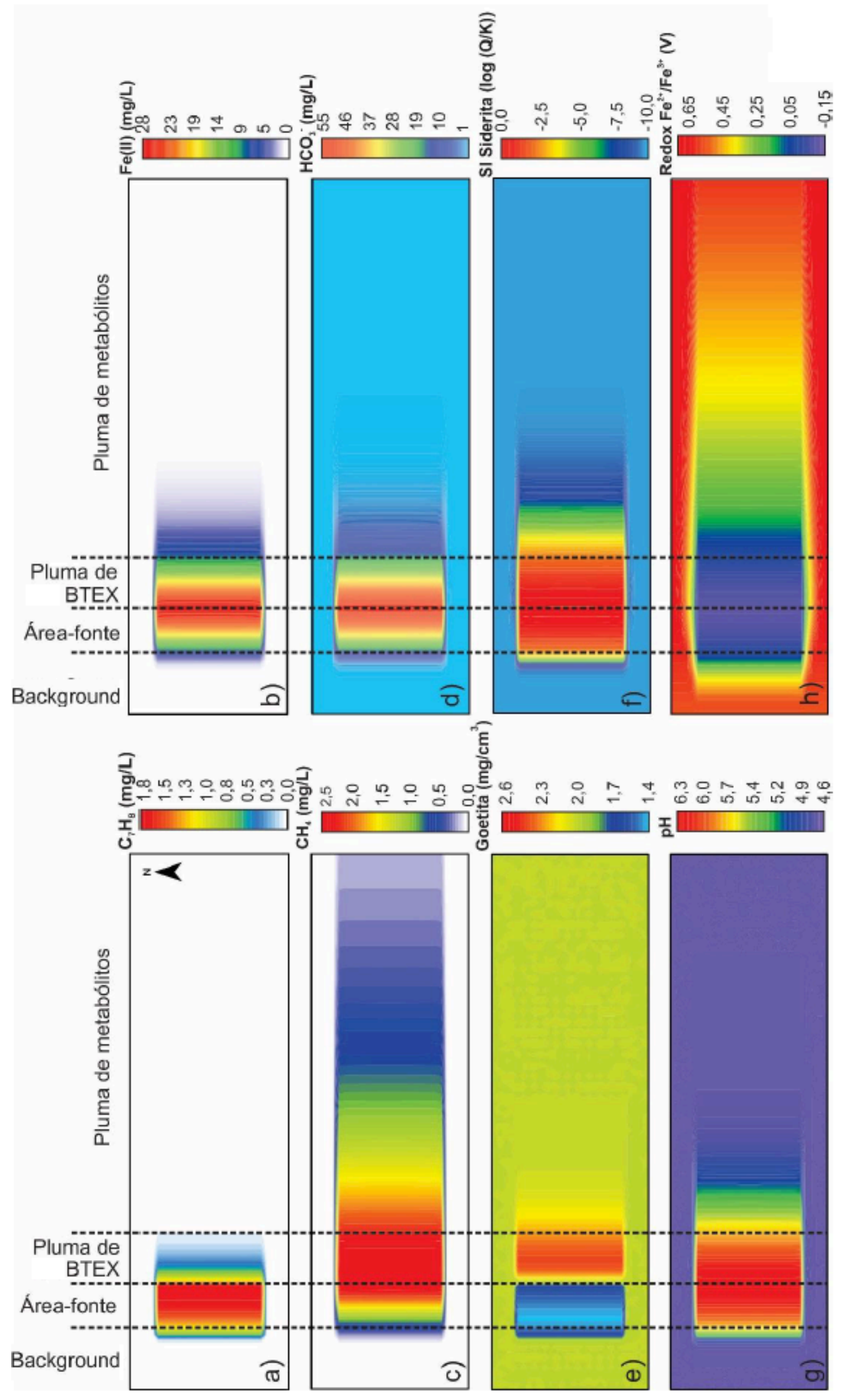

Escala Gráfica

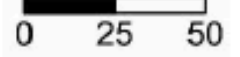

Figura 3

Resultados da simulação: a) Concentração de Tolueno; b) Concentração de $\mathrm{Fe}(\mathrm{II})$; c) Concentração de $\mathrm{CH}_{4}$; d) Concentração de $\mathrm{HCO}_{3}{ }^{-}$e) Massa de goetita no sedimento; d) SI da Siderita; g) $\mathrm{pH}$; h) Potencial redox $\mathrm{Fe}^{3+} / \mathrm{Fe}^{2+}$. 

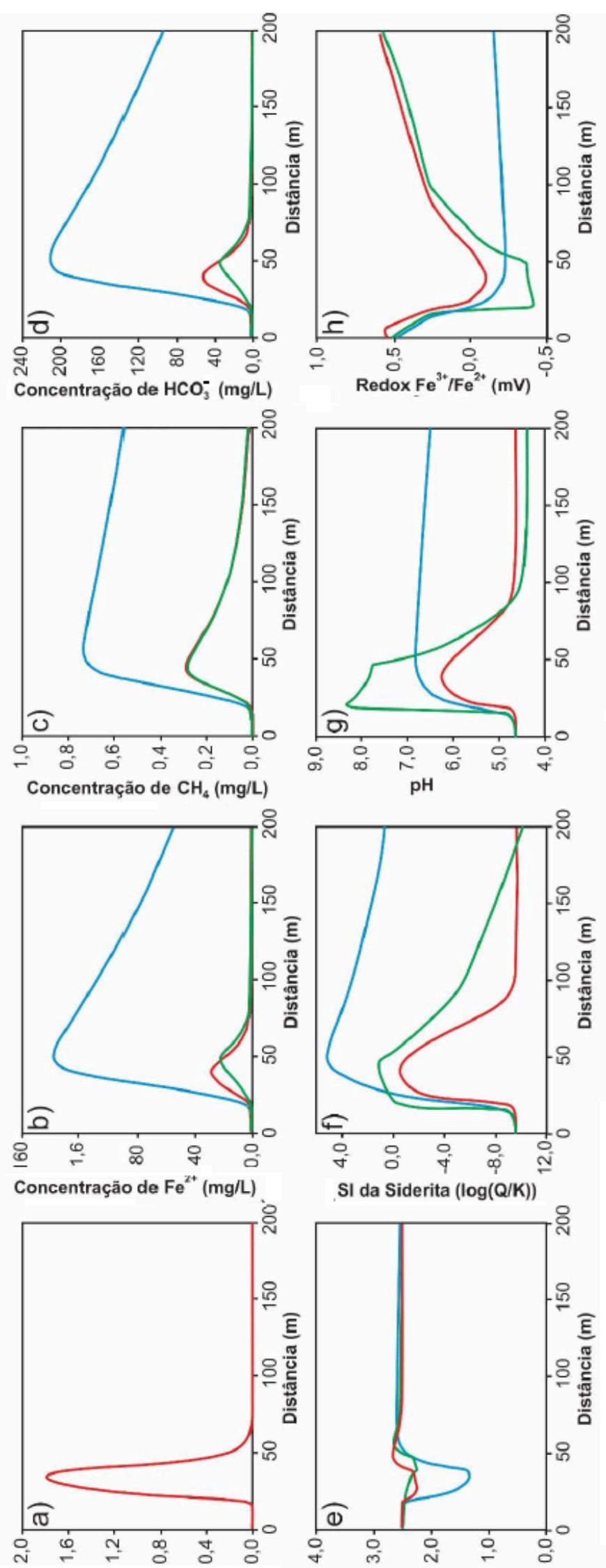

Concentração de tolueno $(\mathrm{mg} / \mathrm{L})$

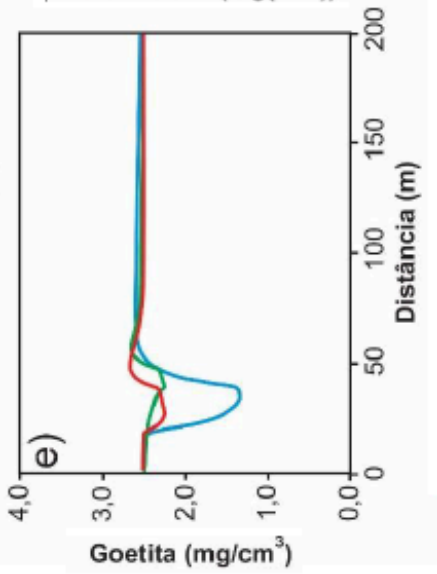

— Cenárin 1 Cẹnárin? Cenário 3

Figura 4

Comparação dos três cenários simulados: a) Concentração de tolueno; b) Concentração de $\mathrm{Fe}(\mathrm{II})$; c) Concentração de $\mathrm{CH}_{4}$; d) Concentração de $\mathrm{HCO}_{3}^{-}$; e) pH; f) IS da siderita; g) Concentração de goetita no sedimento; h) Potencial redox do par 
lisado (Eq. 2), enquanto no Cenário 3, as concentrações são muito elevadas por não ocorrer o consumo de $\mathrm{HCO}_{3}^{-}$na oxidação do $\mathrm{Fe}^{2+}$. Nota-se que os cenários 2 e 3 fornecem valores de SI_siderita muito maiores que o Cenário 1, indicando supersaturação em relação a este mineral. No caso do Cenário 2, a supersaturação da siderita é gerada pelo aumento do $\mathrm{pH}$ (Figura 4g), devido à ausência do efeito tampão da $\mathrm{PCO} 2$. No caso do Cenário 3 , a supersaturação está relacionada à alta concentração de $\mathrm{Fe}^{2+}$ (Figura $4 \mathrm{~b}$ ) e $\mathrm{HCO}_{3}^{-}$ (Figura 4d) na solução, uma vez que a reação da oxidação do $\mathrm{Fe}^{2+}$ (Eq. 4) não é representada neste cenário.

Nota-se que os valores de $\mathrm{pH}$ são mais elevados nos Cenários 2 e 3 em comparação ao Cenário 1. No caso do Cenário 2 , em que as variações de $\mathrm{pH}$ seguem a mesma tendência do Cenário 1 , com maiores valores $(>8)$ dentro da área-fonte e caindo rapidamente a jusante. Este comportamento é decorrente da ausência de ácido carbônico na solução, resultante do equilíbrio sob $\mathrm{PCO}_{2}$ elevada. No caso do Cenário 3, a queda do $\mathrm{pH}$ à jusante da áreafonte é tênue e resulta da ausência da reação de oxidação do $\mathrm{Fe}(\mathrm{II})$, fazendo com que as

\section{DISCUSSÃO}

Em climas tropicais e subtropicais existem condicionantes geoquímicos e hidrológicos particulares, que exigem modelos geoquímicos conceituais relacionados à biodegradação de hidrocarbonetos distintos daqueles elaborados para climas temperados. As características tradicionalmente associadas a climas tropicais são as elevadas temperaturas da água subterrânea. $\mathrm{Na}$ área de estudo, as temperaturas são tipicamente acima de $26{ }^{\circ} \mathrm{C}$ durante todo o ano (TERAMOTO; CHANG, 2019), favorecendo a assimilação microbiana de hidrocarbonetos em subsuperfície. Outra característica que pode ser igualmente associada a climas tropicais é a de óxidos de ferro no sedimento, gerada por processos de laterização. Em razão da abundância de ferro e da disponibilidade limitada de outros aceptores de elétrons $\left(\mathrm{NO}_{3}{ }^{-}\right.$e $\left.\quad \mathrm{SO}_{4}{ }^{2-}\right)$, a redução dissimilatória do ferro é o principal mecanismo de metabolização de hidrocarbonetos em muitos sítios contaminados no Brasil.

Outra característica que pode ser associada a climas tropicais é a elevada amplitude de variação do nível d'água ao longo do ano, induzida por elevadas taxas de precipitação. concentrações de $\mathrm{HCO}_{3}^{-}$se mantenham elevadas.

Similarmente ao $\mathrm{pH}$, o potencial redox do par $\mathrm{Fe}^{3+} / \mathrm{Fe}^{2+}$ (Figura 4h) é distinto nos 3 cenários, com valores menores no Cenário $3 \mathrm{e}$ especialmente no Cenário 2. No Cenário 3 , o Eh do par $\mathrm{Fe}^{3+} / \mathrm{Fe}^{2+}$ está relacionado à alta concentração de $\mathrm{Fe}^{2+}$ (Figura 4b). No caso do Cenário 2, o potencial oxi-redutor de $\mathrm{Fe}^{3+} / \mathrm{Fe}^{2+}$ espelha a curva de $\mathrm{pH}$, concordando com a equação de Nerst (GORSKI et al., 2016), em que o $\mathrm{pH}$ elevado no Cenário 2 (Figura 4g) explica o valor mais negativo do par $\mathrm{Fe}^{3+} / \mathrm{Fe}^{2+}$. Este resultado é apoiado pelos experimentos de Gorski et al. (2016), que demonstraram que os valores de Eh das soluções contendo goetita e $\mathrm{Fe}^{2+}$ são cada vez mais negativos em valores de $\mathrm{pH}$ mais altos.

A depleção em relação à goetita na áreafonte é bastante distinta no Cenário 3, em que é mais acentuada. Esta diferença pode estar associada à reoxidação de parte do ferro férrico nos cenários 1 e 2 . Complementarmente, notase um acúmulo de goetita imediatamente à jusante da área-fonte nos cenários 1 e 2, indicando a precipitação de goetita nestas porções (Figura 4e).

$\mathrm{Na}$ área de estudo, por exemplo, Teramoto e Chang $(2017 ; 2018)$ registraram flutuações do nível d'água superior a 4 metros ao longo dos anos (2002-2015). Em razão da flutuação do nível d'água em patamares elevados, ocorrem condições favoráveis para o trapeamento do LNAPL, como demonstrado por Pede (2009) e Isler et al. (2018). Por esta razão, é esperado que a transferência de massa para a água seja o mecanismo mais efetivo para a depleção do LNAPL. Similarmente ao LNAPL, o ar é igualmente trapeado na zona saturada durante a subida do nível d'água, como demonstrado por diversos trabalhos, incluindo Faybishenko (1995), Williams e Oostrom (2000), Haberer et al. (2012), Marinas et al. (2013) e McLeod et al. (2015). A porção superior da zona saturada contém uma proporção variável de ar trapeado e representa a zona "quase saturada" (FAYBISHENKO, 1995). A presença de ar trapeado pode liberar oxigênio para a água em escala de tempo de décadas, como apontado por McLeod et al. (2015).

A $\mathrm{PCO}_{2}$ é um importante agente que governa processos geoquímicos em subsuperfície por tamponar o $\mathrm{pH}$ das águas 
subterrâneas e controlar o equilíbrio químico das espécies carbonáticas dissolvidas (MACPHERSON, 2009). Além disso, a PCO2 de aquíferos superficiais, tanto na zona saturada quanto nas não saturadas, pode ser de 1 a 2 ordens de grandeza maior quando comparada com a da atmosfera sob condições naturais (BROOK et al., 1983; MACPHERSON, 2009; KESSLER et al., 2011; BORDIGNON et al., 2015; BALDINI et al., 2018; JOUN et al., 2019). Deve ser destacado que a $\mathrm{PCO}_{2}$ é particularmente elevada em ambientes tropicais (BROOK et al., 1983), onde a taxa de respiração do solo é alta. A despeito da grande quantidade de $\mathrm{CO}_{2}$ nas zonas não-saturada e saturada, sua influência é normalmente subestimada nos modelos geoquímicos de áreas contaminadas por hidrocarbonetos.

As simulações realizadas indicam que o modelo conceitual de Teramoto e Chang (2019) consegue reproduzir satisfatoriamente as características geoquímicas do sítio contaminado por querosene de aviação. Para ponderar a importância relativa da $\mathrm{PCO}_{2}$ e das reações secundárias, simulações de três cenários distintos foram realizadas. Notoriamente, os cenários simulados indicaram uma alta discrepância entre si (Figura 3), enfatizando a importância da $\mathrm{PCO}_{2}$ e da oxidação. O Cenário 1 ajusta-se melhor aos dados de campo apresentados por Teramoto e Chang (2019) do que os Cenários 2 ou 3, indicando que tanto a oxigenação quanto o equilíbrio imposto por elevada $\mathrm{PCO}_{2}$ do aquífero são requisitos cruciais para a reprodução das simulações numéricas de transporte reativo em aquíferos contaminados por compostos BTEX em climas tropicais. A concordância entre os dados de campo e os valores simulados de $\mathrm{pH}, \mathrm{HCO}_{3}{ }^{-}$e do índice de saturação da siderida ( $\mathrm{SI}_{\text {siderita }}$ ) confirma que $\mathrm{PCO}_{2}$ elevada desempenha papel importante no controle da hidroquímica do aquífero contaminado com hidrocarbonetos.

A não representação de reações secundárias (Eq. 4 e 5) nas simulações do cenário 2 geram resultados irreais e incompatíveis com os dados de campo, indicando que o trapeamento de ar exerce um papel crucial na evolução redox de sítios contaminados por BTEX. Provavelmente, as concentrações de $\mathrm{Fe}^{2+} \mathrm{e}$ $\mathrm{CH} 4$ na área-fonte (Tabela 1) seriam mais elevadas e o potencial redox mais reduzido se não existisse a liberação de oxigênio do ar trapeado, como indicam as simulações com ausência das reações 4 e 5 (Cenário 3). Cabe destacar que, teoricamente, com base nas relações estequiométricas das Eq. 2 e 3, é possível estimar a massa de compostos BTEX biodegradados a partir das concentrações de $\mathrm{Fe}^{2+}$ e $\mathrm{CH}_{4}$. Entretanto, esta estimativa não oferece resultados realistas, uma vez que parcela significativa do $\mathrm{Fe}^{2+}$ e do $\mathrm{CH}_{4}$ na fase aquosa é perdida em razão da oxidação destas espécies químicas. No modelo, a cinética de oxidação de ferro foi maior que a de redução de ferro (Eq. 4), indicando condições favoráveis para a oxidação do ferro ferroso na área-fonte. No entanto, uma vez que a massa produzida de $\mathrm{Fe}^{2+}$ pela Eq. 2 é maior que a massa removida pela Eq. 4, existe um excedente global de ferro ferroso dentro da área-fonte.

Cabe destacar que a oxidação do $\mathrm{Fe}^{2+}$ é dependente do $\mathrm{pH}$, sendo demonstrado por princípios termodinâmicos (MARTIN, 2005; MORGAN; LAVAH, 2007) e laboratoriais (HABEER et al., 2015) que em soluções com pH abaixo de 6 a oxidação abiótica é pouco significativa. No entanto, Geroni e Sapsford (2011) demonstraram que os valores das taxas de oxidação de $\mathrm{Fe}^{2+}$ medidas no campo são de 1 a 3 ordens de grandeza maiores do que reportado em estudos laboratoriais nas mesmas faixas de $\mathrm{pH}$. Além disso, Katsoyiannis e Zouboulis (2004) destacaram que a oxidação de $\mathrm{Fe}^{2+}$ mediada pelo metabolismo bacteriano é várias ordens de grandeza maior que sua oxidação abiótica. Por esta razão, especula-se aqui que a ação enzimática de bactérias nativas propicie as elevadas cinéticas de oxidação do $\mathrm{Fe}^{2+}$, a despeito dos valores de $\mathrm{pH}$ não serem favoráveis.

Teramoto e Chang (2019) verificaram que o $\mathrm{Fe}^{2+}$ e $\mathrm{CH}_{4}$ apresentam fortes variações na concentração, alternando períodos de aumentos e diminuições das concentrações. Tendo em vista que este comportamento coincide com os períodos de subida e descida do nível d'água, os autores interpretaram-no como resultado da oxidação mais eficiente em períodos nos quais o nível d'água sobe e, consequentemente, ocorre trapeamento do ar. Por esta razão, as concentrações medidas de BTEX e subprodutos são transitórias e, deste modo, as simulações numéricas aqui apresentadas reproduzem um período específico. Assim, assume-se que as cinéticas das reações apresentadas serão distintas na calibração de modelos em períodos distintos.

Os resultados da concentração de goetita no sedimento do aquífero (Figura 4e) mostram 
acúmulo pronunciado imediatamente à jusante da área-fonte, em resposta à oxidação de ferro ferroso (Eq. 4). Esse processo é semelhante à observação de campo de Tuccillo et al. (1999) no site de Bemidji, no qual $70 \%$ do ferro ferroso produzido por mineralização de hidrocarbonetos é oxidado na zona de transição anóxica/óxica. Apesar da produção de ferrihidrita, na simulação foi produzida

\section{CONCLUSÕES}

Este trabalho reforça a importância de se compreender as condicionantes geoquímicas relacionadas à biodegradação de BTEX em aquíferos tropicais e subtropicais. As simulações numéricas permitiram avaliar que o modelo conceitual proposto por Teramoto e Chang (2019) é capaz de reproduzir as condições geoquímicas de campo, onde climas tropicais e subtropicais atendem às condições hidrológicas e geoquímicas que afetam o com-

\section{REFERÊNCIAS}

BALDINI J. U., BERTRAM R. A.; RIDLEY H. E. 2018. Ground air: A first approximation of the Earth's second largest reservoir of carbon dioxide gas. Science of the Total Environment, 616, 1007-1013.

BETHKE C.M.; YEAKEL S. 2016. The Geochemist's Workbench Reference Manual, Release 11.0. Manual do Usuário. Aqueous Solutions, LLC.

BORDIGNON, R.; TERAMOTO, E. H.; CHANG, H. K.; HESPANHOL, E. C. B. 2015. Caracterização isotópica de $\mathrm{CO}_{2}$ dissolvido em águas subterrâneas em área contaminada por querosene de aviação, município de Paulínia (SP). Águas Subterrâneas, 29(3), 301-314.

BROOK G. A., FOLKOFF M. E.; BOX E. O. 1983. A world model of soil carbon dioxide. Earth Surface Processes and Landforms, 8(1), 79-88.

COLOMBANI N., MASTROCICCO M., GARGINI A., DAVIS G. B., \& Prommer H. 2009. Modelling the fate of styrene in a mixed petroleum hydrocarbon plume. Journal of contaminant hydrology, 105(1-2), 38-55.

DAS S., HENDRY M. J., \& ESSILFIE-DUGHAN J. 2010. Transformation of two-line ferrihydrite to goethite and hematite as a function of $\mathrm{pH}$ and temperature. Environmental science \& technology, 45(1), 268-275.

DECLERCQ I., CAPPUYNS, V., \& DUCLOS Y. 2012. Monitored natural attenuation (MNA) of contaminated soils: state of the art in Europe-a critical evaluation. Science of the Total Environment, 426, 393-405.

FAYBISHENKO B. A. 1995. Hydraulic behavior goetita, uma vez que a ferrihidrita é rapidamente convertida para goetita, uma fase termodinamicamente mais estável. A transformação da ferrihidrita é uma reação dependente do $\mathrm{pH}$ e da temperatura e ocorre rapidamente, como demonstrado nos experimentos de Schwertmann e Murad (1983) e Das et al. (2010).

portamento de compostos orgânicos nas águas subterrâneas. Ressalta-se, neste contexto, que os resultados obtidos sugerem que as condições de campo observadas não podem ser explicadas sem o equilíbrio de alta $\mathrm{PCO} 2 \mathrm{em}$ um sistema aberto em relação ao $\mathrm{CO} 2$ e sem a oxigenação do aquífero mediada pela difusão do oxigênio a partir do ar trapeado na zona saturada.

of quasi-saturated soils in the presence of entrapped air: Laboratory experiments. Water Resources Research, 31(10), 2421-2435.

GERONI J. N., \& SAPSFORD D. J. 2011. Kinetics of iron (II) oxidation determined in the field. Applied geochemistry, 26(8), 1452-1457.

GORSKI C. A., EDWARDS, R., SANDER, M., Hofstetter, T. B., \& Stewart, S. M. 2016. Thermodynamic characterization of iron oxideaqueous $\mathrm{Fe}^{2+}$ redox couples. Environmental science \& technology, 50(16), 8538-8547.

HABERER C. M., ROLLE, M., CIRPKA O. A., \& GRATHWOHL, P. 2012. Oxygen transfer in a fluctuating capillary fringe. Vadose Zone Journal, 11(3).

HABERER C. M., MUNIRUZZAMAN, M., GRATHWOHL P., \& ROLLE M. 2015. Diffusive-dispersive and reactive fronts in porous media: Iron (ii) oxidation at the unsaturated-saturated interface. Vadose Zone Journal, 14(5).

HUNTER K. S., WANG, Y., \& van CAPPELLEN P. 1998. Kinetic modeling of microbially-driven redox chemistry of subsurface environments: coupling transport, microbial metabolism and geochemistry. Journal of hydrology, 209(1-4), 53-80.

ISLER E., TERAMOTO, E. H., BAESSA M. P. M., Pede M. A. Z., \& Kiang, C. H. 2018. Trapeamento de LNAPL observado por meio da técnica de fluorescência induzida por laser (LIF). Águas Subterrâneas, 32(3), 315-324.

JOUN, W. T., ROSSABI, J., SHIN, W. J., \& LEE, K. K. 2019. Real-time multi-level $\mathrm{CO}_{2}$ 
concentration monitoring in vadose zone wells and the implication for detecting leakage events. Journal of environmental management, 237, 534-544.

KATSOYIANNIS I. A., \& ZOUBOULIS A. I. 2004. Biological treatment of $\mathrm{Mn}$ (II) and Fe (II) containing groundwater: kinetic considerations and product characterization. Water research, 38(7), 1922- 1932.

KESSLER T. J., \& HARVEY C. F. 2001. The global flux of carbon dioxide into groundwater. Geophysical research letters, 28(2), 279-282.

LOVLEY D. R., BAEDECKER M. J., LONERGAN, D. J., COZZARELLI, I. M., PHILLIPS, E. J., \& SIEGEL, D. I. 1989. Oxidation of aromatic contaminants coupled to microbial iron reduction. Nature, 339(6222), 297.

MACPHERSON, G. L. 2009. $\mathrm{CO}_{2}$ distribution in groundwater and the impact of groundwater extraction on the global $\mathrm{C}$ cycle. Chemical Geology, 264(1-4), 328-336.

MARINAS M., ROY J. W. \& SMITH J. E. 2013. Changes in entrapped gas content and hydraulic conductivity with pressure. Groundwater, 51(1), 41-50.

MARTIN, S. T. 2005. Precipitation and dissolution of iron and manganese oxides. Environmental Catalysis, 61-81.

MAYER K. U., FRIND E. O., \& BLOWES D. W. 2002. Multicomponent reactive transport modeling in variably saturated porous media using a generalized formulation for kinetically controlled reactions. Water Resources Research, 38(9), 13-1.

MCLEOD H. C., ROY J. W., \& SMITH J. E. 2015. Patterns of entrapped air dissolution in a twodimensional pilot-scale synthetic aquifer. Groundwater, 53(2), 271-281.

MILlER, C. T., POIRIER-MCNEIL, M. M., \& MAYER, A. S. 1990. Dissolution of trapped nonaqueous phase liquids: Mass transfer characteristics. Water Resources Research, 26(11), 2783-2796.

MILES B., Peter A., \& TEUTSCH G. 2008. Multicomponent simulations of contrasting redox environments at an LNAPL site. Groundwater, 46(5), 727-742.

MORGAN B., \& LAHAV O. 2007. The effect of $\mathrm{pH}$ on the kinetics of spontaneous $\mathrm{Fe}$ (II) oxidation by $\mathrm{O}_{2}$ in aqueous solution-basic principles and a simple heuristic description. Chemosphere, 68(11), 2080-2084.

NATIONAL RESEARCH COUNCIL. 2000.
Natural attenuation for groundwater remediation. National Academies Press.

NG G. H. C., BEKINS B. A., COZZARELLI I. M., BAEDECKER M. J., BENNETT, P. C., \& AMOS, R. T. 2014. A mass balance approach to investigating geochemical controls on secondary water quality impacts at a crude oil spill site near Bemidji, MN. Journal of contaminant hydrology, 164, 1-15.

PEDE M. A. Z. 2009. Flutuação do lençol freático e sua implicação na recuperação de hidrocarbonetos: um estudo de caso. Instituto de Geociências e Ciências Exatas, Universidade Estadual Paulista, Rio Claro, $141 \mathrm{p}$.

PROMMER, H., DAVIS, G. B., \& BARRY, D. A. 1999. Geochemical changes during biodegradation of petroleum hydrocarbons: field investigations and biogeochemical modelling. Organic Geochemistry, 30(6), 423-435.

SCHREIBER M. E., CAREY G. R., FEINSTEIN D. T., \& BAHR J. M. 2004. Mechanisms of electron acceptor utilization: implications for simulating anaerobic biodegradation. Journal of contaminant hydrology, 73(1-4), 99-127.

SCHWERTMANN, U. \& MURAD E. 1983. Effect of $\mathrm{pH}$ on the formation of goethite and hematite from ferrihydrite. Clays and Clay Minerals, 31(4), 277-284.

TERAMOTO E. H., \& CHANG H. K. 2017. Field data and numerical simulation of btex concentration trends under water table fluctuations: Example of a jet fuel-contaminated site in Brazil. Journal of contaminant hydrology, 198, 37-47.

TERAMOTO E. H., \& CHANG H. K. 2018. Métodos WTF e simulação numérica de fluxo para estimativa de recarga-exemplo Aquífero Rio Claro em Paulínia/SP. Águas Subterrâneas, 32(2), 173-180.

TERAMOTO E. H., \& CHANG H. K. 2019. Geochemical conceptual model of BTEX biodegradation in an iron-rich aquifer. Applied Geochemistry, 100, 293-304.

TUCCILlO M. E., COZZARELli I. M., \& Herman J. S. (1999). Iron reduction in the sediments of a hydrocarbon-contaminated aquifer. Applied Geochemistry, 14(5), 655-667.

WIEDEMEIER, T. H., RIFAI H. S., NEWELL C. J., \& WILSON J. T. (1999). Natural attenuation of fuels and chlorinated solvents in the subsurface. John Wiley \& Sons.

WILLIAMS M. D., \& OOSTROM M. (2000). Oxygenation of anoxic water in a fluctuating water table system: an experimental and numerical study. Journal of Hydrology, 230(12), $70-85$ 